\title{
Efeito do Tratamento Alcalino de Fibras de Juta no Comportamento Mecânico de Compósitos de Matriz Epóxi
}

\author{
Eduardo N. Pires \\ Instituto Federal de Educação, Ciência e Tecnologia de Santa Catarina, IF-SC \\ Claudia Merlini, Hazim Ali Al-Qureshi, Gean V. Salmória, Guilherme M. O. Barra \\ Departamento de Engenharia Mecânica, UFSC
}

\begin{abstract}
Resumo: Neste trabalho foi avaliada a influência do tratamento químico de fibras vegetais nas propriedades mecânicas e dinâmico-mecânicas de resina epóxi reforçada com tecidos de fibras de juta. As fibras foram modificadas a partir de solução de hidróxido de sódio, e caracterizadas por microscopia eletrônica de varredura (MEV), espectrometria no infravermelho com transformada de Fourier (FTIR) e resistência à tração. As análises de FTIR e MEV evidenciaram, respectivamente, que o tratamento alcalino promoveu a remoção parcial da hemicelulose e modificou a morfologia das fibras de juta. Amostras dos compósitos com fibras tratadas e não tratadas foram confeccionadas a partir da laminação manual seguida da moldagem por compressão. Compósitos com fibras tratadas apresentaram propriedades mecânicas e módulo de armazenamento maiores do que as da resina epóxi sem reforço e compósitos com fibras sem tratamento. As micrografias de microscopia eletrônica de varredura (MEV) revelaram que compósitos com fibras tratadas possuem menor quantidade de vazios e menor quantidade de pull-out, sugerindo maior adesão com a matriz polimérica do que os compósitos com fibras sem tratamento.
\end{abstract}

Palavras-chave: Compósito polimérico, resina epóxi, fibra de juta, adesão fibra-matriz, tratamento químico.

\section{The Influence of Alkaline Treatment on Jute Fiber- Reinforced Epoxy Resin Composite}

Abstract: This study was aimed at evaluating the efficiency of chemical treatment on the dynamic mechanical and mechanical properties of composite materials with jute fibers and epoxy matrix resin. The surfaces of the jute fibers were modified by contact with sodium hydroxide solutions, and characterized through scanning electron microscopy (SEM), Fourier transform infrared spectroscopy (FTIR) and tensile strength testing. The FTIR and SEM analysis evidenced that the alkaline treatment promoted removal of the hemicellulose and modification in the morphology of the jute fibers, respectively. Polymeric composites with untreated and treated jute fiber were prepared through hand lay-up process followed by compression molding. The treated jute fiber composites displayed higher mechanical properties and storage modulus values than the epoxy resin and untreated fiber composites. The scanning electron micrographs (SEM) revealed that treated jute fiber composites have less voids and pull-out, suggesting a better adhesion with the polymeric matrix than untreated fiber composites.

Keywords: Polymeric composites, epoxy resin, jute fibers, adhesion fiber-matrix, chemical treatment.

\section{Introdução}

A investigação de materiais de fontes naturais e renováveis para aplicações em produtos vêm se intensificando nos últimos anos. A conscientização dos consumidores da preservação ambiental e constantes mobilizações de órgãos que atuam em defesa do meio ambiente vêm despertando a atenção de autoridades, que começam a legislar em prol do desenvolvimento sustentável. Somado a este fato, o alto grau de competitividade hoje existente em um mercado globalizado gera uma necessidade cada vez maior nas indústrias para o desenvolvimento de produtos de baixo custo e mais leves.

Neste contexto, existe um interesse crescente na utilização de materiais lignocelulósicos (fibras de juta, sisal, coco, banana e curauá) como reforço em compósitos de matrizes poliméricas. O interesse da utilização das fibras vegetais está relacionado ao seu baixo custo, densidade menor do que as fibras de vidro, além de serem oriundas de fontes renováveis, não abrasivas ${ }^{[1]}$, biodegradáveis $^{[2,3]}$ e disponíveis em território nacional. Dentre as fibras vegetais, a fibra de juta é uma das mais usadas como reforço de matrizes termoplásticas e termofixas por seu baixo custo e propriedades mecânicas adequadas ${ }^{[4]}$. Somado a este fator, no Brasil, a cultura de juta (Corchorus Capsularis) tornou-se uma das principais atividades econômicas da população ribeirinha da região amazônica, sendo fundamental para a fixação de milhares de famílias ao campo. A juta pode ser plantada nas calhas dos rios, não provocando desmatamento, sendo que seu ciclo de plantio leva seis meses e coincide com os períodos de cheia e vazante dos rios. Devido à importância econômica regional desse tipo de cultura e da grande produção nacional, sua utilização em compósitos poliméricos mostra-se como uma alternativa economicamente viável e tem sido amplamente estudada por vários grupos de pesquisa $^{[4]}$.

Entretanto, uma das dificuldades para o uso de fibras de juta e outras fibras vegetais como agente de reforço em matrizes poliméricas, de forma a obterem-se compósitos com propriedades mecânicas e estabilidade dimensional desejadas, é a fraca adesão interfacial com o polímero. A interface fibra-matriz desempenha um papel importante nas propriedades mecânicas e físicas dos compósitos poliméricos, pois é através da interface que ocorre a transferência de tensões da matriz para as fibras ${ }^{[5,6]}$. Portanto, se as interações entre as fases forem fracas, dificilmente será obtido um aumento das propriedades mecânicas do polímero a ser modificado ${ }^{[7]}$. Uma maneira de contornar este problema é modificar a estrutura da fibra através de métodos químicos ou 
físicos. Um dos métodos mais utilizados é o tratamento alcalino, que extrai a lignina e a hemicelulose, resultando em mudanças na estrutura, na morfologia, nas dimensões e nas propriedades mecânicas das fibras ${ }^{[5,8]}$. É importante destacar que além da região interfacial, as propriedades dos compósitos com fibras vegetais são também funções das propriedades das fases constituintes, das suas quantidades relativas e da geometria das fibras utilizadas como agente de reforço ${ }^{[5]}$.

Dentre os polímeros termofixos, a resina epóxi representa uma das classes mais versáteis, apresentando uma combinação singular de propriedades, como resistência à tração e tenacidade, resistência química, à umidade e à corrosão, boas propriedades térmicas, adesivas e elétricas. Isto geralmente não é encontrado em outros materiais poliméricos, sendo sua aplicação atrativa quando se deseja produzir materiais de engenharia. Além disso, os grupos reativos epóxi podem reagir com as hidroxilas presentes nas fibras de juta, resultando em uma adesão adequada entre fibra/resina epóxi, e consequentemente contribuir para o aumento das propriedades mecânicas da resina a ser reforçada.

Visando o aproveitamento de recursos naturais disponíveis em território nacional e buscando-se alternativas que sejam economicamente viáveis para o reforço de matrizes poliméricas, utilizaram-se fibras de juta para a produção de compósitos com resina epóxi. O efeito do tratamento químico na superfície das fibras de juta e nas propriedades mecânicas dos compósitos de matriz de resina epóxi foi avaliado através dos ensaios de tração, compressão e dinâmico-mecânicos.

\section{Experimental}

\section{Material e métodos}

A resina epóxi utilizada foi a TCR 550 e o agente de cura XR-435, ambos fabricados pela empresa Triepox e fornecidos pela Central do Fiberglass. O tecido de fibra de juta foi adquirido junto à Companhia Têxtil de Castanhal.

O tratamento alcalino das fibras foi realizado utilizando-se uma solução de hidróxido de sódio (5\% em massa), à temperatura ambiente por 6 horas. Posteriormente, as fibras foram lavadas até $\mathrm{pH}$ neutro e secas em estufa a $60{ }^{\circ} \mathrm{C}$ até obtenção de massa constante.

A resina foi preparada misturando-se a resina com o agente de cura numa proporção em massa de $2: 1$, respectivamente. Inicialmente, a resina foi colocada em um balão volumétrico, sob vácuo, com agitação constante de $30 \mathrm{rpm}$ e aquecida a $50{ }^{\circ} \mathrm{C}$. Posteriormente, a resina foi resfriada e o agente de cura foi adicionado, mantendo-se sob agitação. Após cinco minutos de mistura, o material foi vertido em moldes com formatos apropriados para serem submetidos aos ensaios de flexão, tração e dinâmico-mecânico.

Os compósitos contendo duas camadas de tecido de fibras de juta impregnadas por resina epóxi foram inicialmente moldados por laminação manual sobre uma chapa de aço galvanizado. Em seguida o compósito foi submetido a uma pressão de $2 \mathrm{~N} \cdot \mathrm{mm}^{-2} \mathrm{em}$ prensa hidráulica, por 24 horas. Posteriormente, foi realizada a pós-cura do compósito em uma estufa a $100{ }^{\circ} \mathrm{C}$ por 90 minutos.

\section{Caracterização}

Os espectros de infravermelho das fibras de juta foram obtidos em um espectrofotômetro Perkin-Elmer, na faixa de $4000 \mathrm{~cm}^{-1}$ a $500 \mathrm{~cm}^{-1}$, utilizando-se pastilhas de Brometo de Potássio.

O ensaio de tração das fibras de juta, da resina epóxi e dos compósitos foi realizado de acordo com as normas ASTM D3822, ASTM D638 e ASTM D3039M, respectivamente, em uma Máquina Universal de Ensaios, marca EMIC, modelo DL2000. A velocidade de ensaio utilizada foi de $3 \mathrm{~mm} / \mathrm{min}$ e as células de carga utilizadas foram de $500 \mathrm{~N}$ para as fibras e de $20 \mathrm{kN}$ para a resina e compósitos, respectivamente.

O ensaio de flexão em três pontos foi realizado para a resina e para os compósitos conforme norma ASTM D790, com $30 \mathrm{~mm}$ entre os pontos e velocidade de ensaio de $5 \mathrm{~mm} / \mathrm{min}$.

Microscopia Eletrônica de Varredura foi utilizada para avaliar a superfície das fibras de juta e a superfície de fratura dos compósitos, utilizando-se um microscópio da marca Jeol, modelo JSM-6390LV.

A análise dinâmico mecânica (DMA) foi realizada com amostras de $35 \times 5 \times 1,4 \mathrm{~mm}$, em um equipamento TA DMA800, a uma freqüência de $1 \mathrm{~Hz}$, com deformação relativa à área útil igual a $0,6 \%$ e taxa de aquecimento de $3{ }^{\circ} \mathrm{C} / \mathrm{min}$.

\section{Resultados e Discussões}

A Figura 1 apresenta os espectros de infravermelho das fibras de juta sem tratamento e com tratamento alcalino. O espectro das fibras de juta sem tratamento é caracterizado por uma banda em $3418 \mathrm{~cm}^{-1}$, referente ao estiramento vibracional dos grupos $\mathrm{OH}$ presentes na celulose $^{[9]}$. As bandas em 2921 e $2847 \mathrm{~cm}^{-1}$ estão relacionadas

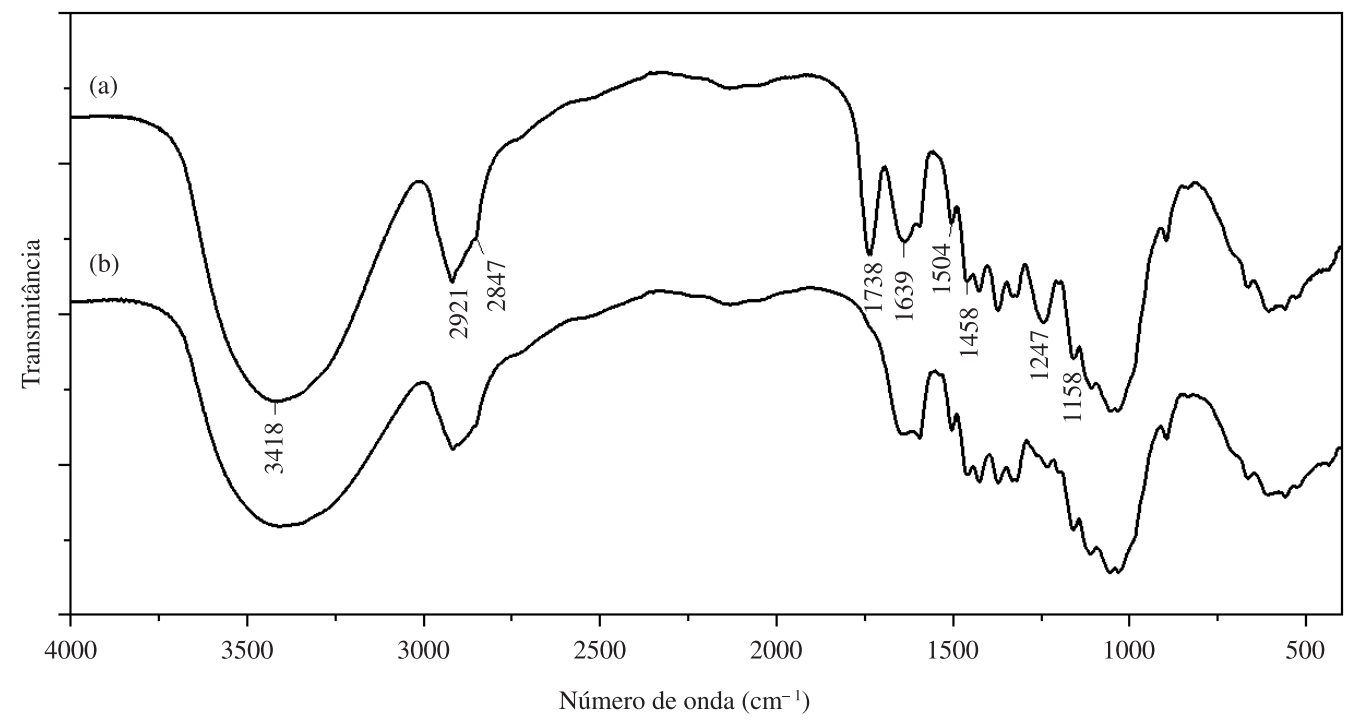

Figura 1. Espectros de infravermelho das fibras de juta (a) sem tratamento e (b) com tratamento alcalino. 


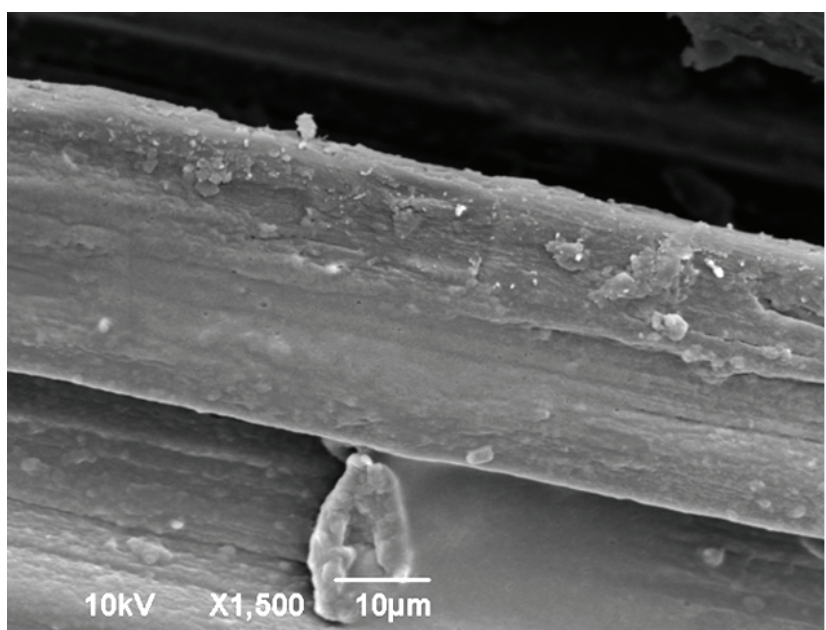

(a)

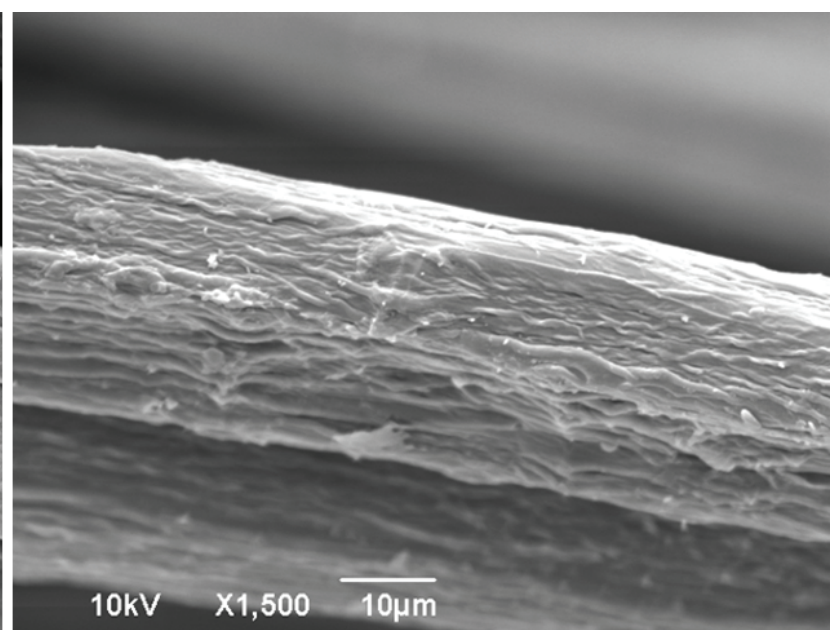

(b)

Figura 2. Micrografias superficiais das fibras de juta (a) sem tratamento e (b) com tratamento alcalino.

Tabela 1. Valores da tensão na ruptura, módulo elástico, deformação e diâmetro das fibras de juta não tratadas e tratadas com $\mathrm{NaOH}$.

\begin{tabular}{|c|c|c|}
\hline Propriedade & $\begin{array}{c}\text { Fibra de juta não } \\
\text { tratada (ST) }\end{array}$ & $\begin{array}{c}\text { Fibra de juta } \\
\text { tratada } \mathrm{NaOH}(\mathrm{N})\end{array}$ \\
\hline $\begin{array}{l}\text { Tensão de ruptura } \\
\text { (MPa) }\end{array}$ & $71,07 \pm 16,27$ & $70,17 \pm 13,64$ \\
\hline Deformação (\%) & $1,04 \pm 0,39$ & $1,24 \pm 0,25$ \\
\hline $\begin{array}{l}\text { Módulo elástico } \\
\text { (MPa) }\end{array}$ & $9362 \pm 3352$ & $9586 \pm 3617$ \\
\hline Diâmetro $(\mu \mathrm{m})$ & $59,39 \pm 20,78$ & $48,17 \pm 14,1$ \\
\hline
\end{tabular}

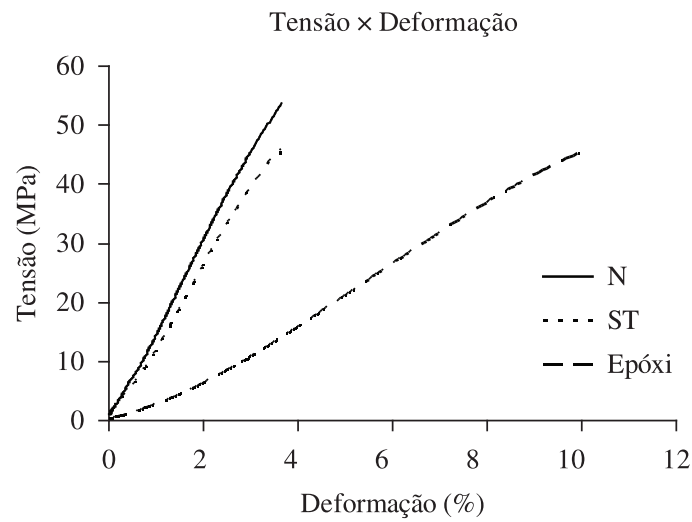

Figura 3. Curvas representativas de resistência a tração da resina epóxi e dos compósitos com fibras sem tratamento (ST) e tratadas com $\mathrm{NaOH}(\mathrm{N})$.

ao estiramento $\mathrm{C}-\mathrm{H}$ alifático dos grupos metila $\left(\mathrm{CH}_{3}\right)$ e metileno $\left(\mathrm{CH}_{2}\right)$, respectivamente ${ }^{[10]}$. Em 1738 e $1639 \mathrm{~cm}^{-1}$ são observadas as bandas referentes ao estiramento dos grupos carbonila presentes na hemicelulose e lignina, respectivamente ${ }^{[11,12]}$. As bandas em 1504, 1458 e $1158 \mathrm{~cm}^{-1}$ estão relacionadas ao estiramento da ligação C-C no anel aromático, à deformação assimétrica do grupo metila e ao grupo C-O-C da celulose, respectivamente. A banda em $1247 \mathrm{~cm}^{-1}$ é atribuída ao estiramento dos grupos acetil da hemicelulose ${ }^{[9,10]}$.

As fibras de juta tratadas apresentam espectro similar, porém não são observadas as bandas em 1738 e $1247 \mathrm{~cm}^{-1}$, atribuídas ao estiramento dos grupos $\mathrm{C}=\mathrm{O}$ e acetil da hemicelulose, respectivamente. Essas alterações estão relacionadas ao fato de que a hemicelulose é solúvel mesmo a baixas concentrações de álcalis, fazendo com que a mesma seja parcialmente removida da fibra, confirmando a modificação química ${ }^{[12]}$.
Tensão na Ruptura - tração

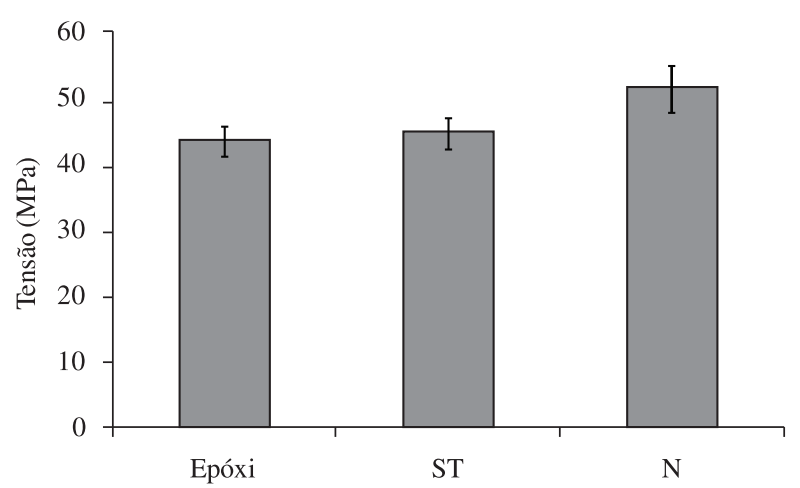

(a)

Módulo elástico - tração

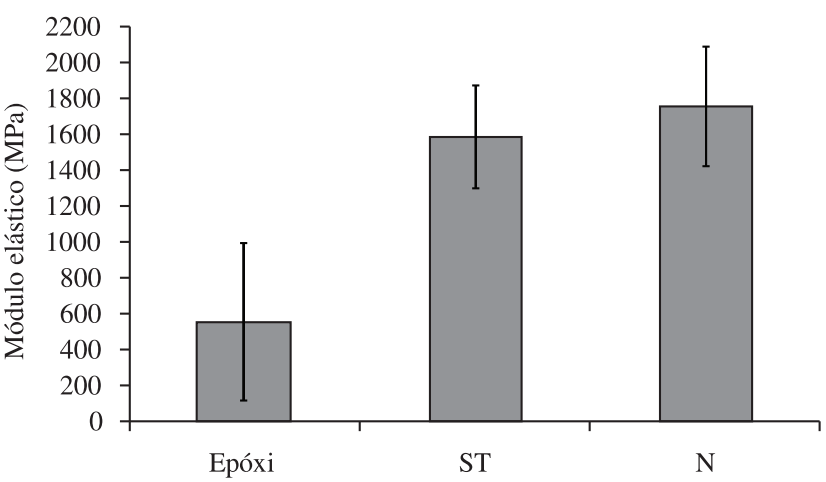

(b)

Figura 4. Gráficos de (a) tensão na ruptura e (b) módulo elástico, obtidos no ensaio de tração para resina epóxi e compósitos com fibra de juta sem tratamento (ST) e tratadas com hidróxido de sódio $(\mathrm{N})$.

As micrografias da superfície das fibras de juta sem tratamento e tratadas, mostradas na Figura 2, sugerem que o tratamento alcalino aumentou a rugosidade das fibras, provavelmente devido à retirada da hemicelulose e lignina. De acordo com Silva ${ }^{[13]}$, as modificações observadas na superfície das fibras podem resultar em maior efetividade de difusão da matriz polimérica nas regiões interfibrilares, levando a um aumento da adesão interfacial entre fibra e matriz. 
Observa-se a partir da Tabela 1 que o tratamento não exerceu influência significativa nos valores de tensão na ruptura, módulo de elasticidade e deformação das fibras de juta. Verificou-se também uma redução de até $19 \%$ no diâmetro médio das fibras tratadas em relação as sem tratamento, devido à remoção da hemicelulose decorrente do tratamento. Gassan et al. ${ }^{[14]}$ também verificaram uma redução de $28 \%$ no diâmetro de fibras de jutas tratadas com solução de $\mathrm{NaOH}$ com concentração de $28 \%$ por 30 minutos. Entretanto, outros estudos sobre o tratamento alcalino de fibras de juta mostraram que a remoção da lignina e celulose afeta as propriedades mecânicas das fibras. Nos estudos realizados por Sinhá et al. ${ }^{[10]}$ observaram-se, aumentos de $33 \%, 50 \%$ e $78 \%$ no módulo elástico após tempos de tratamento alcalino de 2,4 e 8 horas, respectivamente. Segundo Bledzki et al. ${ }^{[5]}$ e Sinhá et al. ${ }^{[10]}$ a

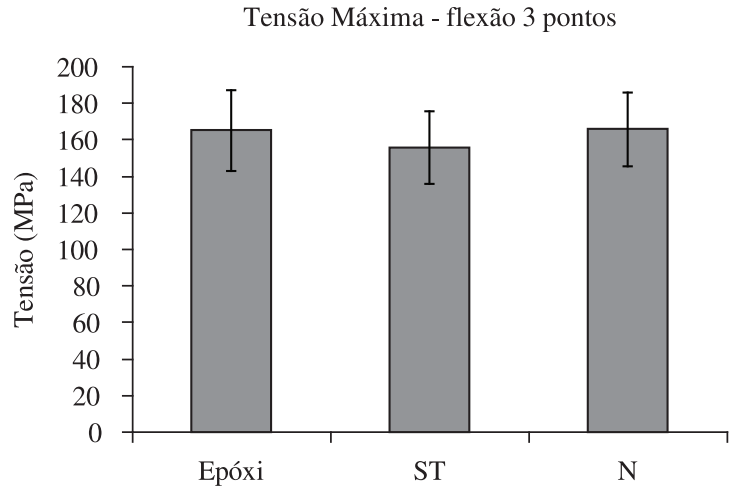

(a)
Módulo Elástico - flexão 3 pontos

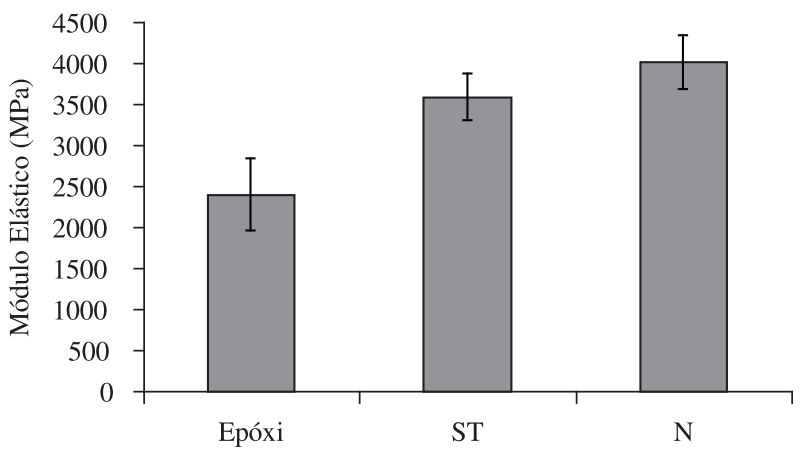

(b)

Figura 5. Gráficos de (a) tensão máxima e (b) módulo elástico, obtidos do ensaio de flexão para resina epóxi e compósitos com fibra de juta sem tratamento (ST) e tratadas com hidróxido de sódio $(\mathrm{N})$.

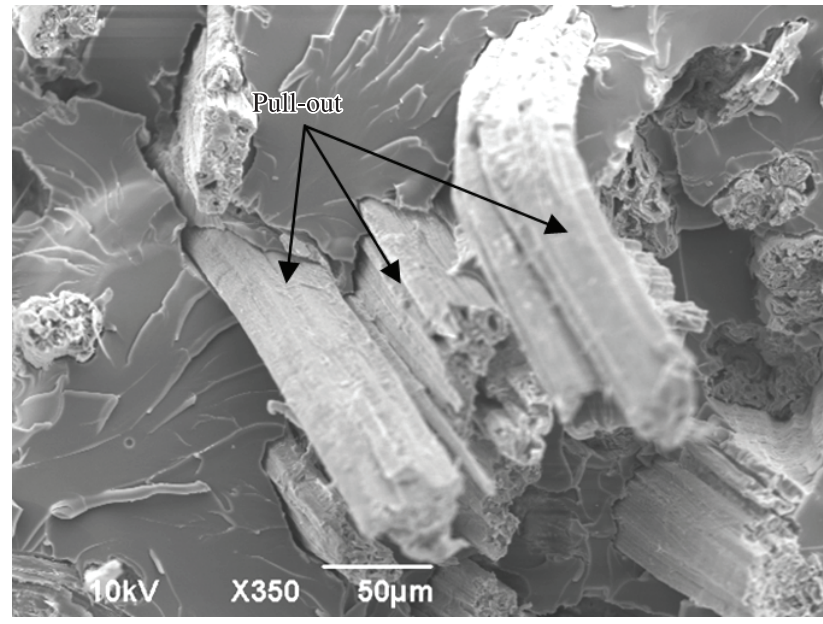

(a)

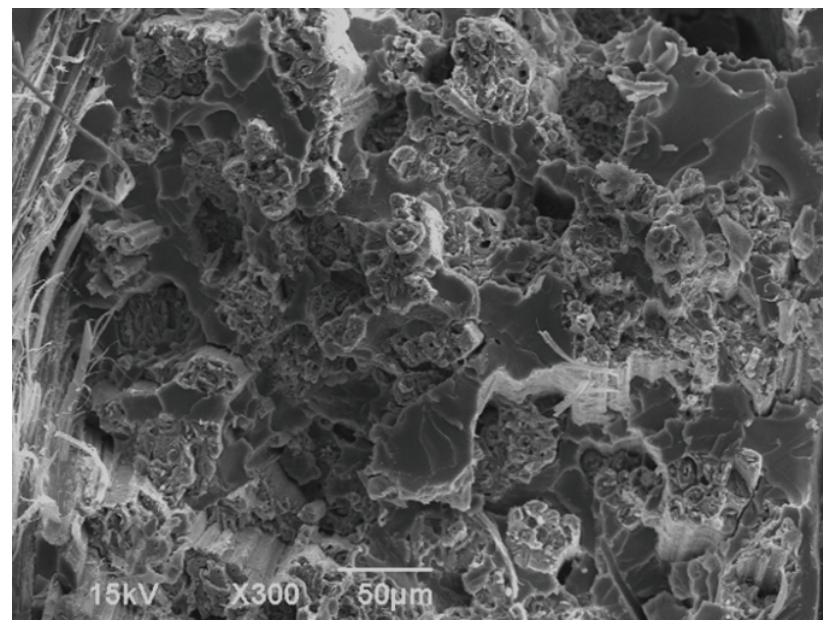

(c)

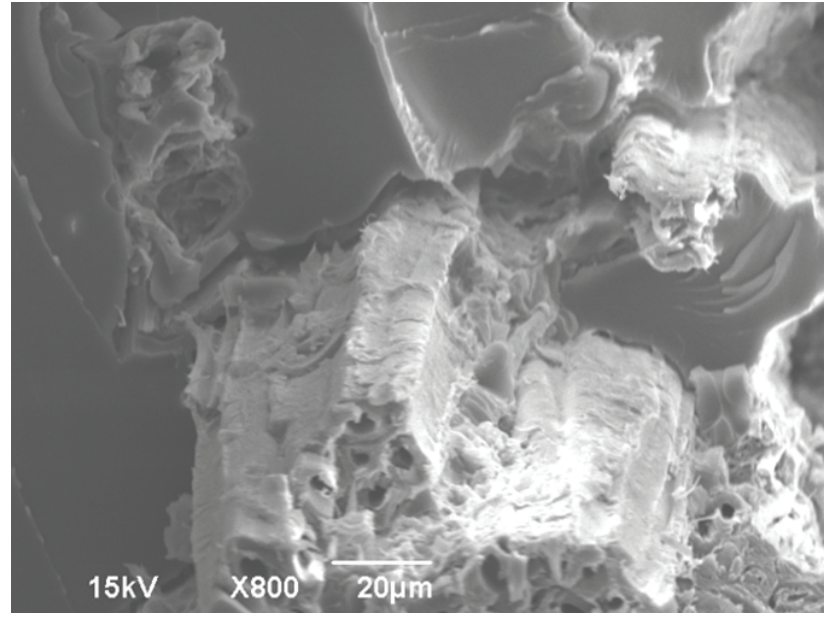

(b)

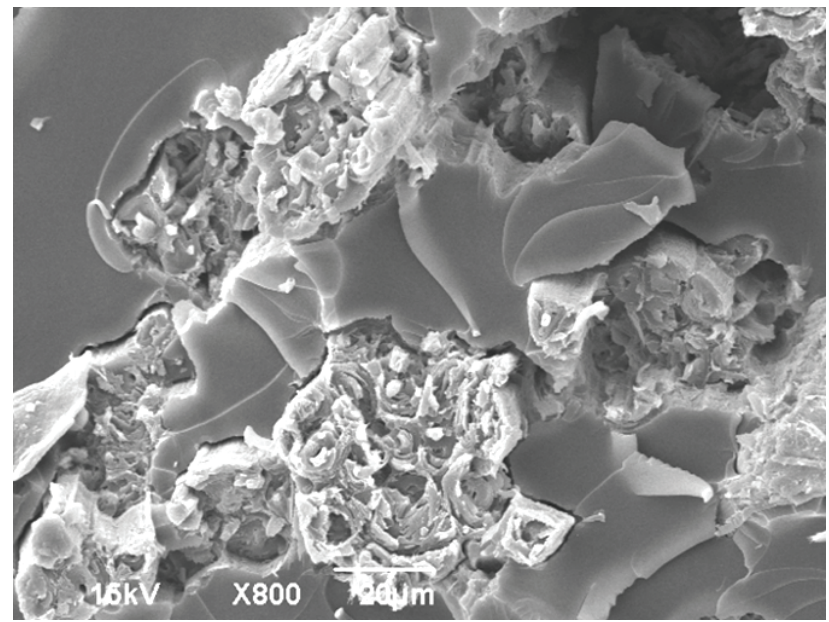

(d)

Figura 6. MEV dos compósitos juta/epóxi do ensaio de tração: (a) e (b) Compósito com fibras sem tratamento e (c) e (d) com fibras tratadas com hidróxido de sódio. 
mudança nas propriedades mecânicas das fibras, principalmente no módulo elástico, está relacionada ao fato de que com a remoção da hemicelulose e da lignina, que atuam como material de cementação separando as cadeias de celulose, as fibrilas têm maior facilidade de ser rearranjar, permitindo um melhor empacotamento das cadeias de celulose e maior orientação molecular, induzindo um aumento no grau de cristalinidade. Além disso, segundo Gassan et al. ${ }^{[14]}$ também ocorre a formação de novas ligações de hidrogênio entre as cadeias de celulose como resultado da remoção da lignina e da hemicelulose.

Os valores das propriedades mecânicas das fibras de juta apresentaram alta dispersão, uma vez que a estrutura e propriedades das fibras vegetais variam consideravelmente em função das condições ambientais, de cultivo e processamento ${ }^{[9]}$.

As curvas de tensão versus deformação da resina epóxi e dos compósitos com fração volumétrica de $30 \%$ de fibras tratadas e não tratadas estão apresentadas na Figura 3. Os valores de tensão na ruptura dos compósitos com fibras sem tratamento são semelhantes aos da resina sem fibras, porém o compósito com fibras tratadas apresentaram um aumento da tensão na ruptura de $18 \%$ em relação à resina epóxi (Figura 4). Este fato comprova que com o tratamento alcalino há uma maior eficiência no mecanismo de transferência de tensão da matriz para as fibras, induzindo assim maior resistência à tração. Pode-se inferir que o aumento da rugosidade superficial somado à maior concentração de grupos $\mathrm{OH}$ livres (provenientes da celulose) promove maior adesão mecânica e química ${ }^{[16]}$.

A deformação na ruptura diminui com a adição das fibras de juta, tanto para os compósitos com fibras sem tratamento como para os compósitos com fibras tratadas, conforme observado na Figura 3. Por outro lado, nota-se um aumento no módulo elástico de 185 e $216 \%$ para os compósitos com fibras de juta sem tratamento e com fibras tratadas, respectivamente, em relação à matriz de resina epóxi, conforme observado na Figura 4. Esse comportamento está relacionado à incorporação das fibras que possuem maior rigidez e menor deformação do que a matriz ${ }^{[9,15]}$.

Na Figura 5 estão apresentados os gráficos de tensão máxima e módulo elástico, obtidos a partir do ensaio de flexão a três pontos. Pode-se verificar que os valores de tensão máxima dos compósitos com fibras de juta sem tratamento e tratadas são similares aos da resina epóxi. No entanto, o módulo de elasticidade a flexão dos compósitos com fibras tratadas e não tratadas é substancialmente maior do que o da resina epóxi.

A Figura 6 apresenta as micrografias da superfície de fratura dos ensaios de tração dos compósitos com fibras sem tratamento e com fibras tratadas. A partir das micrografias dos compósitos

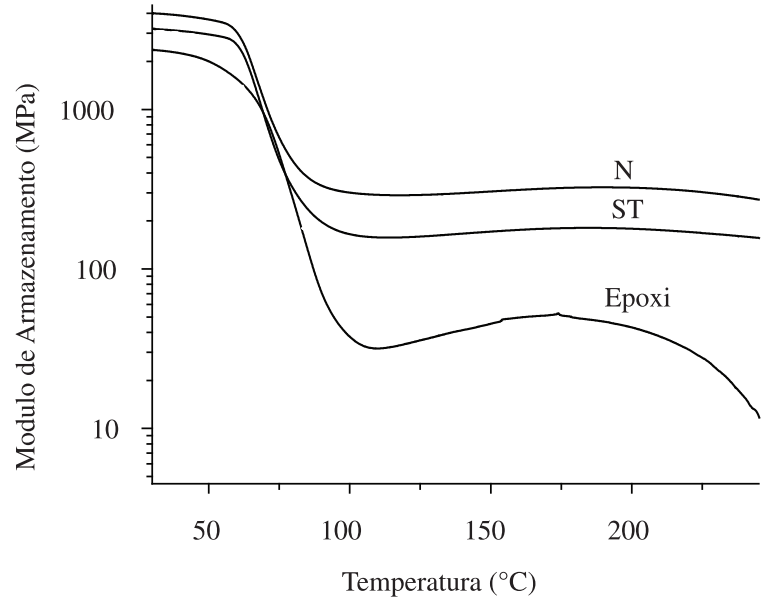

(a)

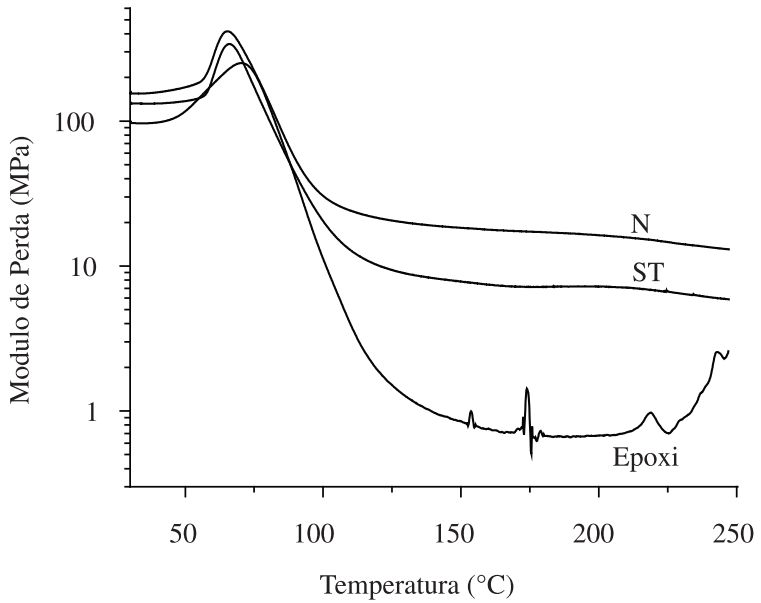

(b)

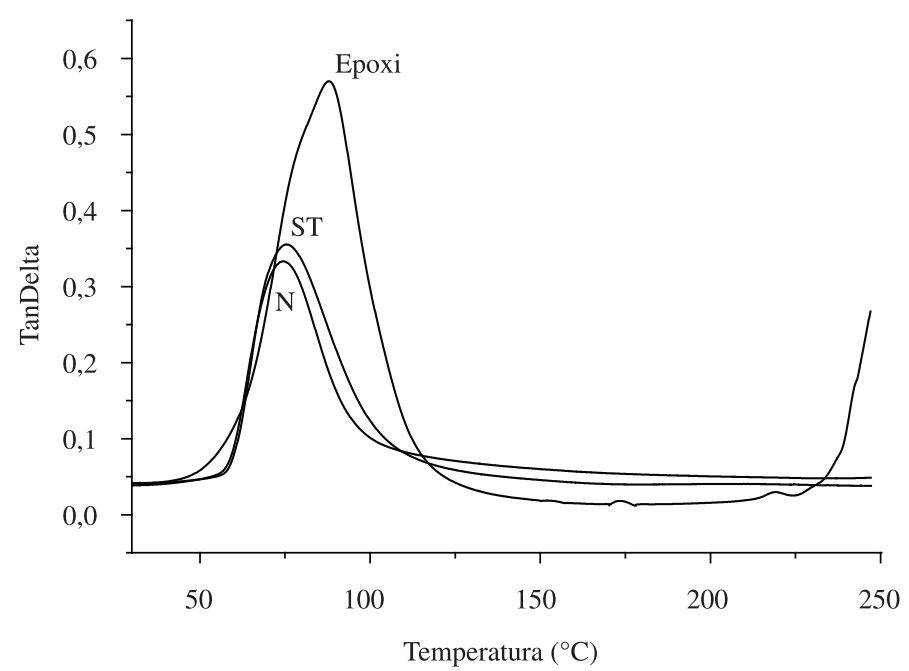

(c)

Figura 7. a) Módulo de armazenamento, b) Módulo de perda e c) tan $\delta$, em função da temperatura, para a resina epóxi e compósitos com fibra de juta sem tratamento (ST) e tratada com hidróxido de sódio $(\mathrm{N})$. 
com fibras sem tratamento verifica-se maior quantidade de fibras arrancadas da matriz, pull-out (Figura 6a), e cavidades entre a fibra e a matriz (Figura 6b), indicando menor adesão interfacial do que nos compósitos com fibras de juta submetidas ao tratamento alcalino (Figuras 6c, d). Estas observações corroboram com os resultados dos ensaios mecânicos apresentados anteriormente, uma vez que os compósitos com fibras tratadas apresentaram maiores valores de módulo de tração e flexão, e tensão na ruptura do que os compósitos com fibras sem tratamento.

As curvas do módulo de armazenamento (E'), módulo de perda (E") e $\tan \delta$ para a resina epóxi e para os compósitos com fibras sem tratamento e tratadas estão apresentados na Figura 7. A Figura 7a mostra a variação do módulo de armazenamento (E') em função da temperatura, em que se verifica um aumento do módulo de armazenamento e também uma redução do valor da tangente de perda (relação do módulo de perda e módulo de armazenamento E'/E') com a adição das fibras (Figura 7c), devido a restrição da mobilidade das cadeias localizadas ${ }^{[17]}$, promovendo um material com maior rigidez. Além disso, é possível observar a diminuição da temperatura de transição vítrea dos compósitos estudados quando comparados com a resina epóxi. O decréscimo da Tg da matriz dos compósitos pode ser atribuído à umidade absorvida pelas fibras ou, no caso dos compósitos com fibras tratadas, devido ao resíduo de hidróxido de sódio do tratamento (grupos hidroxila), que atuam como agente plastificante. $\mathrm{O}$ mesmo comportamento foi observado para compósitos híbridos de matriz epóxi e compósitos de resina de poliéster insaturado reforçada com pó de madeira ${ }^{[18,19]}$.

\section{Conclusão}

A modificação química das fibras de juta com tratamento alcalino foi evidenciada pela técnica de infravermelho, na qual foi possível detectar o desaparecimento das bandas de absorção do grupo carbonila e acetil, devido à remoção da hemicelulose. Além disso, verificaram-se alterações nas características superficiais das fibras a partir da análise das micrografias de microscopia eletrônica de varredura. Compósitos com fibras sem tratamento apresentaram valores de tensão na ruptura similares às da resina epóxi pura, não atuando como agente de reforço. Sendo assim, para aplicações não estruturais, estas fibras passam a ser uma boa alternativa de matéria-prima para atuar como carga de enchimento. No entanto, compósitos com fibras tratadas apresentaram aumento nas propriedades mecânicas em relação à resina sem reforço e aos compósitos com fibras sem tratamento. Conclui-se que os tecidos de fibras de juta podem ser utilizados como agente de reforço em matriz de resina epóxi, desde que seja realizado um tratamento químico das fibras com o objetivo de aumentar a adesão entre as fibras e a matriz polimérica.

\section{Agradecimentos}

Ao Conselho Nacional de Pesquisa (CNPq) e a Coordenação de Aperfeiçoamento de Pessoal de Nível Superior (CAPES).

\section{Referências Bibliográficas}

1. Iozzi, M. A.; Martins, G. S.; Martins, M. A.; Ferreira, F. C.; Job, A. E. \& Mattoso, L. H. C. - Polímeros, 20, p.25 (2010).
2. Megiatto Junior, J. D. - "Fibras de Sisal: Estudo de propriedades e modificações químicas visando à aplicação em compósitos de matriz fenólica". Tese de Doutorado, Universidade de São Paulo, Brasil (2006).

3. Becker, D.; Kleinschmidt, A. C.; Balzer, P. S. \& Soldi, V. - Polímeros, 21, n.1, p.7 (2011). http://dx.doi.org/10.1590/S0104-14282011005000012

4. Albuquerque Neto, J. R.; Carvalho, L. H. \& Araújo, E. M. - Polímeros, 17, n.1, p.10 (2007). http://dx.doi.org/10.1590/ S0104-14282007000100006

5. Merlini, C.; Soldi,V. \& Barra, G. M. O. - Polym. Test., 30, p.833 (2011). http://dx.doi.org/10.1016/j.polymertesting.2011.08.008

6. Bledzki, A. K. \& Gassan, J. - Prog. Polym. Sci., 24, p.221 (1999). http://dx.doi.org/10.1016/S0079-6700(98)00018-5

7. Joseph, S.; Sreekala, J. M. S.; Oommen, Z.; Koshyc, P. \& Thomas, S. - Compos. sci. technol., 62, p.1857 (2002). http://dx.doi.org/10.1016/ S0266-3538(02)00098-2

8. Mohanty, A. K.; Misra, M. \& Drzal, L. T. - "Natural fibers, biopolymers, and biocomposites". Taylor \& Francis, Boca Raton (2005). http:// dx.doi.org/10.1201/9780203508206

9. Merlini, C. - "Análise experimental de compósitos de poliuretano derivado de óleo de mamona e fibras de bananeira", Dissertação de mestrado, Universidade Federal de Santa Catarina, Brasil (2011).

10. Sinha, E. \& Rout, S. K. - J. Mater. Sci., 43, p.2590 (2008). http://dx.doi. org/10.1007/s10853-008-2478-4

11. Guimarães, J. L.; Frollini, E.; Silva, C. G.; Wypych, F. \& Satyanarayana, K. G. Ind. Crop Prod., 30, p.407 (2009). http://dx.doi.org/10.1016/j. indcrop.2009.07.013

12. Corrales, F.; Vilaseca, F.; Llop, M.; Gironès, J.; Mendéz, J. A. \& Mutjé P. J. Hazard. Mater., 144, p.730 (2007). PMid:17320283. http://dx.doi. org/10.1016/j.jhazmat.2007.01.103

13. Silva, R. V. - "Compósito de resina poliuretana derivada do óleo de mamona e fibras vegetais". Tese de Doutorado, Universidade de São Paulo, Brasil (2003).

14. Gassan, J. \& Bledzki, A. K. Compos. Sci. technol., 59, p.1303 (1999). http://dx.doi.org/10.1016/S0266-3538(98)00169-9

15. Sanchez, E. M. S.; Cavani, C. S.; Leal, C. V. \& Sachez, C. G. Polímeros, 20, n.3, p.194 (2010). http://dx.doi.org/10.1590/S010414282010005000034

16. Vilaseca, F.; Mendéz, J. A.; Pèlach, A.; Llop, M.; Cañigueral, N.; Gironès, J.; Turon, X. \& Mutjè. P. Process Biochem., 42, p.329 (2007). http://dx.doi.org/10.1016/j.procbio.2006.09.004

17. Ray,D.; Sarkar, B.K.; Das, S. \& Rana,A.K. - Compos. Sci. tecnol., 62,911 (2002). http://dx.doi.org/10.1016/S0266-3538(02)00005-2

18. Dutra, R. C. L. - Polymer, Oxford, 41, n.10, p.3841 (2000). http:// dx.doi.org/10.1016/S0032-3861(99)00552-2

19. Marcovich, N. E., Aranguren, M. I. \& Reboredo, M. M. - Polymer, Oxford, 42, n.2, p.815 (2001). http://dx.doi.org/10.1016/S00323861(00)00286-X 